\title{
El lenguaje en niños con pérdida auditiva prelocutiva que utilizan implante coclear $y$ en niños oyentes
}

\author{
María Paula Brandon \& Fiorella Sobrino \\ Centro Peruano de Audición, Lenguaje y Aprendizaje
}

Recibido: 18 de abril del 2013 / Aprobado: 27 de junio del 2013

El estudio tiene como finalidad determinar si existen diferencias entre el lenguaje de un grupo de niños con pérdida auditiva que utilizan implante coclear desde temprana edad y niños oyentes del mismo rango etario y similares características socioculturales. Para la recolección de datos se utilizaron las pruebas batería objetiva y criterial (bloc screening) y el subtest fonético fonológico de la prueba de exploración del lenguaje comprensivo y expresivo (ELCE). Para ello se evaluó a un grupo de 10 niños con pérdida auditiva prelocutiva y 30 niños oyentes, de entre 7 y 10 años de edad. El muestreo fue intencional. Los resultados demostraron que existen diferencias significativas entre los dos grupos, siendo sustancialmente superior el desarrollo del lenguaje de los niños oyentes, evidenciando mayores diferencias en los componentes léxico semántico y morfosintáctico.

lenguaje / pérdida auditiva / implante coclear

The language development in children with prelocutive hearing loss that use cochlear implant and children with normal hearing

The study aims to determine if there are differences between the language of a group of children with hearing loss who use cochlear implants at an early age and hearing children, the same age range and similar cultural characteristics. For data collection we used Battery Objective and Criterial (BLOC) screening test and Phonological Phonetic Comprehensive Exploration and expressive language (ELCE). We evaluated a group of 10 children with prelingual hearing loss and 30 hearing children between 7 and 10 years of age. Sampling was intentional. The results showed that significant differences do exist between the two groups, with significantly higher language development of hearing children.

language / hearing loss / cochlear implant

Correo electrónico: icpe@cpal.edu.pe 


\section{INTRODUCCIÓN}

La audición es la capacidad de reconocer por la vía auditiva mensajes verbales. En el niño sordo, esta habilidad auditiva se desarrollará poco a poco, ampliando cada vez más su capacidad discriminativa y podrá en un momento dado utilizar su audición en forma eficaz en situaciones más amplias con algunas limitaciones. Una forma de tratar de superar esta limitación es mediante la realización de implantes cocleares en los niños sordos.

Todo niño implantado pasa por un proceso de rehabilitación, bajo una terapia auditivo-oral con la finalidad de minimizar los retrasos en la adquisición del lenguaje y aprendizaje en general. Se sabe que el implante coclear no constituye una cura ni tampoco conlleva a la restauración de la audición a niveles normales, y que niños con igual o distinto tipo de pérdida auditiva no respondían de la misma manera a la metodología oral después de ser implantados, por ello se investigó acerca del nivel de lenguaje que presenta un determinado grupo de niños implantados tras cinco años de implantación aproximadamente, ya que las fuentes plantean ciertas expectativas tras ciertos periodos de implantación, influenciadas por características propias de cada individuo, como el grado y el tipo de pérdida auditiva, la edad auditiva, el nivel de coeficiente intelectual, el apoyo de la familia, entre otras. Por ello se plantea la siguiente pregunta de investigación: ¿existen diferencias significativas entre el lenguaje de niños con pérdida prelocutiva que utilizan implante coclear y el lenguaje de niños oyentes?

\section{LENGUAJE}

Para Saussure (Martínez, 1998) el lenguaje es multiforme y heterociclo, a la vez psíquico, físico y fisiológico, pertenece además al dominio individual y al dominio social, no se deja clasificar en ninguna de las categorías de los hechos humanos, porque no se sabe cómo desarrollar su unidad; mientras que Bloom (Aguado, 1995) sostiene que el lenguaje es la más definitoria actividad psicológica que el hombre realiza según su condición, aparece desde el primer año de vida y acompaña a casi todas nuestras actividades. Se adquiere aparentemente sin esfuerzo y en general nos trae una excesiva atención, pues como Sapir plantea, el lenguaje es una facultad humana no instintiva para comunicar ideas, emociones y deseos por medio de un sistema de símbolos producidos de manera deliberada, los cuales son, sobre todo, símbolos auditivos producidos por los órganos del habla, en tanto que Rondal (Acosta \& Moreno, 1999) define el lenguaje como un repertorio de palabras organizadas en enunciados sobre una base asociativa, instrumento de comunicación donde se destaca no solo la estructuración de los enunciados sino también el uso de estos en diferentes contextos. Para Narbona y Chevrie-Muller (2001) la aparición 
del lenguaje es un tema que está lejos de ser resuelto, pero plantea que distintos «precursores de éste», tales como la atención selectiva, la percepción categórica de los sonidos del habla y la recepción auditiva del habla es lo que hacen que se dé esta adquisición normal. Posteriormente, Owens (Acosta \& Moreno, 1999) lo definió como un código compartido socialmente, un sistema convencional para la representación de conceptos mediante el uso de símbolos arbitrarios y reglas que gobiernan la combinación de esos símbolos, por lo cual se considera que es un sistema compuesto por unidades (signos lingüísticos) que mantienen una organización interna de carácter formal, su uso permite formas singulares de relación y acción sobre el medio social que se materializa en formas concretas de conducta. Finalmente, es definido como un fenómeno homogéneo, que posee un conjunto de componentes relacionados como la percepción y la producción de sonidos articulados propios de cada idioma (fonemas); que implican varios lexemas y dispositivos morfosintácticos que sirven para expresar en un idioma determinado los dispositivos pragmáticos cuya finalidad es la de optimizar la transmisión de información entre el emisor y el receptor y las interacciones entre interlocutores en la comunicación y la organización discursiva, que rige los conjuntos de frases que constituyen los párrafos y los discursos desde un punto de vista principalmente informativo.
Después de analizar las distintas definiciones podemos decir que el lenguaje es la capacidad humana de poder comunicarse usando signos, un mecanismo simbólico a través del cual las personas pueden establecer relaciones y compartir el conocimiento que adquieren por medio de su desarrollo cognitivo, es la principal herramienta de acceso a la vida social y al aprendizaje.

\section{Desarrollo del lenguaje}

Castañeda (1999) sostiene que el desarrollo del lenguaje se divide en dos etapas principales. La primera es la etapa prelingüística, a la que se denomina también como la etapa preverbal; comprende los primeros 10 a 12 meses de edad; es decir, el primer año de vida. Se caracteriza por la expresión bucofonatoria, que de por sí tiene un valor comunicativo; es la fase en la cual el niño establece comunicación con su medio (familia). Es considerada, además, como la etapa del nivel fónico puro, debido a que el infante emite solo sonidos onomatopéyicos; de allí que para estimularlo lingüísticamente se debe utilizar, junto con el lenguaje afectivo y gestual, el lenguaje verbal. La palabra debe acompañar siempre al gesto y las actividades que el niño realice y que hasta hace poco despertaba escaso interés de los especialistas, pero gracias a las investigaciones actuales hoy sabemos que tiene un valor relevante y trascendental en la configuración de las bases del desarrollo lingüístico, puesto que tanto 
las expresiones vocales (sonidos o grupo de sonidos de simple significación) como las expresiones verbales (sonidos, grupo de sonidos, palabras aisladas, etcétera) influyen de modo determinante en el desarrollo posterior de la comunicación lingüística del niño. Esta etapa comprende, a su vez, subetapas o estadios con características particulares que van de acuerdo con la secuencia cronológica del desarrollo integral del niño. La segunda es la etapa lingüística; se inicia con la expresión de la primera palabra, sin embargo, no se puede decir con precisión cuándo comienza, cuándo este anuncio del lenguaje se precisa y confirma, ni cuándo se puede hablar de la «primera palabra». Hay que señalar, además, que las niñas empiezan a hablar un poco antes que los niños. Por otro lado, aparte del sexo, tomando como referencia las peculiaridades individuales, un niño puede demorarse más que otros en una etapa y pasar rápidamente por otra, condicionando la aparición de la primera palabra en los niños en cronologías distintas.

No obstante, los diferentes especialistas estiman que la mayoría de los niños, tal vez el $90 \%$ de ellos, dicen sus primeras palabras cuando tienen entre 15 y 18 meses, aunque esta afirmación no es exacta o concluyente por las razones antes expuestas, por eso la etapa lingüística se considera en forma un tanto amplia, desde aproximadamente el duodécimo mes (un año de edad), pasando el niño de las variadísimas emisiones fónicas del periodo prelingüístico a la adquisición de fonemas propiamente dichos en el plano fonológico (articulaciones fonemáticas), perfeccionándose también el aspecto semántico y sintáctico de las palabras a medida que el niño crece.

Según Acosta y Moreno (1999), en el componente fonético fonológico del lenguaje encontramos la fonética, que trata de las características de los sistemas articulatorio y auditivo del ser humano. Es considerada una disciplina lingüística que estudia cómo se producen y se perciben los sonidos del habla. La fonética precede a la fonología, recoge información sobre la capacidad humana de emitir y percibir sonidos, toma como eje central a la función, estableciendo que el desarrollo fonológico es el proceso de adquisición fonológica que comienza desde el nacimiento con la emisión de los primeros sonidos y continúa de manera progresiva y gradual hasta los cuatro años aproximadamente, momento en que la mayoría de los sonidos aparecen discriminados en palabras simples. El proceso se termina de completar a la edad de los 6 o 7 años, cuando se dominan determinadas estructuras silábicas de consonantes.

En cuanto al componente morfosintáctico, este se ocupa tanto de la descripción de la estructura interna de las palabras como de las reglas de combinación de los sintagmas en oraciones; además, estudia las reglas que intervie- 
nen en la formación de las palabras y de las posibles combinaciones de estas en el interior de las oraciones en las que se estructura una lengua. Describe las reglas de ordenamiento y funcionamiento tanto de las unidades morfológicas como de las sintácticas, incluye el estudio de las unidades morfológicas (morfemas y palabras) y de las sintácticas (sintagmas y oraciones).

La palabra es toda unidad lingüística que con contenido léxico o gramatical puede ser segmentada en el discurso; se puede descomponer en constituyentes más pequeños que se denominan lexemas (unidades específicamente semánticas) y morfemas.

El componente léxico semántico se centra en el estudio del significado de las palabras y de sus combinaciones. Une las palabras entre sí, dando como resultado una oración, la cual se irá complementando conforme a las reglas, ocupándose del estudio del significado de los signos lingüísticos y de sus posibles combinaciones en los diferentes niveles de organización: en palabras, en frases, en los enunciados y en el discurso. Abarca los procesos de codificación y descodificación de los significados del lenguaje. Receptivamente implica la comprensión del lenguaje; es decir, extraer el significado a partir de nuestro sistema simbólico; expresivamente supone una selección apropiada del vocabulario y estructura del lenguaje para transferir significado, lo cual depende del contenido y propósito de lo que se quiera comunicar.

\section{PÉRDIDA AUDITIVA}

Para Davis y Silverman (1971), el término sordera estaba relacionado con un impedimento auditivo total o casi total de la persona, haciendo una distinción con el término hipoacusia, que para ellos implicaba una pérdida leve o parcial de la audición. Ellos hacen referencia a esta distinción ya que opinan que los problemas psicológicos y médicos de una persona sorda no son iguales a los de una persona hipoacúsica, por lo que consideran que no se les debe agrupar indiscriminadamente. Mientras que para Berrocal (s/f), la discapacidad del niño sordo se ubica a nivel de la lengua oral o lengua fónica, porque no puede organizar un sistema lingüístico a partir de lo que dicen los otros. Al no discriminar los sonidos del habla y tener la percepción auditiva dañada, no está en condiciones de transmitir al cerebro los datos lingüísticos acústicos suficientes para el procesamiento de la información, por lo que el desarrollo del lenguaje se ve afectado.

A partir de lo mencionado se puede afirmar que la pérdida auditiva es la disminución o incapacidad de percibir los sonidos del habla y del medioambiente. Esto se puede producir por diversas causas y presentarse en distintos grados de severidad. La clasificación de la pérdida auditiva se puede realizar 
bajo diferentes criterios: el grado de la pérdida auditiva, el momento de aparición de la pérdida, la localización de la lesión o según la etiología de la pérdida. Dependiendo de los resultados de una evaluación audiológica, se ubica al paciente en uno de los siguientes grados:

- Pérdida auditiva leve: entre 20 y 40 decibeles (dB). Es posible la percepción global del habla, a excepción de algunos fonemas en determinadas posiciones y de emisiones a intensidad muy baja.

- Pérdida auditiva moderada: entre 40 y $60 \mathrm{~dB}$. Solo es posible la percepción global del habla con una buena adaptación de audífonos.

- Pérdida auditiva severa: entre 60 y $90 \mathrm{~dB}$. La persona afectada puede percibir los elementos suprasegmentales del habla, pero debe completar los elementos que discrimina auditivamente con la lectura labiofacial; ello es posible con la ayuda del audífono y del entrenamiento auditivo.

- Pérdida auditiva profunda: superior a 90 dB. Para la percepción del lenguaje cobran vital importancia las vías vibrátiles y visuales. La correcta amplificación de los audífonos permite, en la mayoría de los casos, el control de la emisión del sujeto y por tanto constituye una aportación importante a la inteligencia de su habla.

La amplificación que el paciente requiere se puede dar mediante el implan- te coclear al que Portillo (Manrique \& Huarte, 2000) define como un aparato electrónico que se coloca en el oído interno durante una cirugía, diseñado para ayudar a pacientes con sordera neurosensorial de severa a profunda, que obtienen muy poco o ningún beneficio de los audífonos convencionales. Los pacientes podrán detectar sonidos del medio ambiente, la mayoría va a entender el lenguaje sin la ayuda de lectura de labios y algunos podrán utilizar el teléfono.

Mortari, Bevilacqua y Alves (2007) estudiaron el desempeño de la audición y el lenguaje oral en niños con pérdida auditiva neurosensorial profunda bilateral prelingual, que usan implante coclear multicanal, por lo que fue evaluada la audición y el lenguaje de 60 niños. Las variables analizadas estadísticamente fueron: los aspectos psicosociales, el estadio cognitivo del niño, el índice de apoyo de la familia y el desempeño de la audición y del lenguaje con el uso del implante coclear, llegando a la conclusión de que el uso del implante coclear en el tratamiento de niños con deficiencia auditiva neurosensorial pre-lingual es altamente efectivo y complejo por la interacción de variables que interfieren en el desempeño del niño implantado. Asimismo, Franco, Tabanez, Bevilacqua y Vuono (2007) también investigaron acerca del tema, coincidiendo en que el lenguaje de los niños que usan implante coclear es semejante al de los niños oyentes de 
5 años de edad para la expresión y semejante al de los niños de 4 años para la comprensión, lo cual determina que la influencia del tiempo de privación sensorial auditiva es significativo. Sin embargo, en la investigación de Szagun (2006), los resultados demostraron que solo 10 de 22 niños se encontraron dentro del desarrollo del lenguaje natural. Más de la mitad de los niños (12 de 22), experimenta un desarrollo que no se asemeja al natural ya que los niños con implante coclear que se nivelaron usaban esas formas de modo correcto y también cometían los errores típicos de la adquisición natural. De modo muy distinto ocurrió con los niños con implante coclear cuyo desarrollo fue muy lento. Estos niños no alcanzaron a producir oraciones completas, usaban muy poco el plural o las terminaciones verbales correctas y los artículos y palabras auxiliares faltaban en ellos casi por completo.

\section{OBJetivos}

\section{Objetivo general}

Comparar el lenguaje de los niños con pérdida auditiva prelocutiva que utilizan implante coclear y los niños oyentes de 7 a 10 años.

\section{Objetivos específicos}

- Determinar las diferencias en el componente fonético fonológico en- tre los niños sordos prelocutivos con implante coclear y los niños oyentes de 7 a 10 años de edad.

- Establecer las diferencias en el componente morfológico entre los niños sordos prelocutivos con implante coclear y los niños oyentes de 7 a 10 años de edad.

- Identificar las diferencias en el componente sintáctico entre los niños sordos prelocutivos con implante coclear y los niños oyentes de 7 a 10 años de edad.

- Determinar las semejanzas en el componente semántico entre los niños sordos prelocutivos con implante coclear y los niños oyentes de 7 a 10 años de edad.

- Identificar las diferencias en el componente pragmático entre los niños sordos prelocutivos con implante coclear y los niños oyentes de 7 a 10 años de edad.

\section{HIPÓTESIS}

\section{Hipótesis general}

Existen diferencias en el lenguaje en niños con pérdida auditiva prelocutiva que utilizan implante coclear y niños oyentes de 7 a 10 años de edad.

\section{Hipótesis específicas}

1) Existen diferencias en el componente fonético fonológico entre los niños con pérdida auditiva prelocu- 
tiva que utilizan implante coclear y los niños oyentes de 7 a 10 años de edad.

2) Existen diferencias en el componente morfológico entre los niños con pérdida auditiva prelocutiva que utilizan implante coclear y los niños oyentes de 7 a 10 años de edad.

3) Existen diferencias en el componente sintáctico entre los niños con pérdida auditiva prelocutiva que utilizan implante coclear y los niños oyentes de 7 a 10 años de edad.

4) Existen diferencias en el componente semántico entre los niños con pérdida auditiva prelocutiva que utilizan implante coclear y los niños oyentes de 7 a 10 años de edad.

5) Existen diferencias en el componente pragmático entre los niños con pérdida auditiva prelocutiva que utilizan implante coclear y los niños oyentes de 7 a 10 años de edad.

\section{MÉTOdo}

\section{Diseño metodológico}

En un primer momento el estudio fue de tipo descriptivo: se recolectó información sobre el nivel de los componentes del lenguaje para describirlo. En un segundo momento fue de tipo comparativo: se puso a prueba una hipótesis de diferencia de niveles de lenguaje entre dos grupos claramente diferenciados de niños con implante coclear y niños oyentes. Por otro lado, el diseño es transeccional, ya que las observaciones realizadas en la muestra de estudios con los instrumentos de recolección de datos se ejecutaron en un solo momento, según el cronograma de la investigación.

\section{Participantes}

La muestra estuvo compuesta por 40 niños, de los cuales 10 fueron niños con pérdida auditiva prelocutiva que utilizan implante coclear, del colegio Fernando Wiese Eslava, y 30 niños oyentes, con edades que oscilan entre los 7 y los 10 años. Una característica importante de los niños con implante coclear es que antes y después de la implantación estuvieron expuestos a una metodología de enseñanza auditiva-oral.

\section{Instrumentos de recolección de datos}

En el proceso de evaluación del nivel de los componentes del lenguaje en ambos grupos de estudio se utilizó la batería de lenguaje objetiva y criterial (BLOC-screening) y el subtest fonético-fonológico de la prueba de exploración del lenguaje comprensivo y expresivo (ELCE) de López Ginés y Redón Díaz, publicado en 1996 en Madrid. La administración se realizó de manera individual a niños de 4 a 7 años, con una duración de 15 a 30 minutos.

En este estudio se tomó como indicador de validez la estrategia de validez de contenido. La prueba de ELCE 
fue sometida a la opinión de cinco expertas, según los criterios de claridad, analizando si las instrucciones están presentadas en un lenguaje apropiado y las imágenes expuestas de forma clara y precisa, de acuerdo con el tema. La objetividad: si las tareas que tiene que realizar el sujeto se expresan en conductas observables y comprobables (emisión de palabras); la organización: si determina la existencia de una organización apropiada en la presentación de las imágenes (suficiencia), analizando si la calidad y la cantidad de imágenes son las adecuadas para la elocución de las palabras; la intencionalidad: si la prueba es adecuada para valorar aspectos relacionados con el tema de estudio, y finalmente la consistencia: si las imágenes corresponden con la normatividad para lo implicado en el estudio.

En cuanto a la batería de lenguaje objetiva y criterial (BLOC-screening), cuyos autores son Miguel Puyuelo, Jordi Renon, Antoni Solanas y Elizabeth Wiig, en el 2002, en Barcelona, es administrada individualmente a niños entre los 5 y los 14 años por un tiempo de 40 a 60 minutos, aproximadamente, evaluando los componentes morfológico, sintáctico, semántico y pragmático. La interpretación es: si el centil es de 70-100 (superior), el alumno domina las habilidades lingüísticas y puede usarlas correctamente; si el centil es de 60-70 (transición), el alumno necesita ayuda para dominar completamente las habili- dades lingüísticas; si llega solo a 30-60 (emergencia), el alumno muestra un dominio muy bajo y necesita ayuda, por lo que es recomendable aplicar la versión completa de la prueba o módulo para perfilar mejor el problema. Finalmente, si el centil es inferior, de 25-30 (alarma), la competencia del lenguaje es muy reducida y es necesario administrar la versión completa de la prueba o módulo para perfilar mejor el aprendizaje.

\section{Procedimiento}

Una vez determinadas las características de la muestra se solicitaron los permisos correspondientes a los padres e instituciones educativas a las cuales pertenecen los niños, para lo cual se les hizo firmar una autorización. La administración de las pruebas fue de forma individual para los dos grupos de niños y se realizó cuidando las consideraciones métricas del caso para que los resultados sean válidos y confiables. La calificación de las respuestas de los instrumentos de estudio se realizó utilizando un software y de forma manual. Se elaboraron las bases de datos en Excel, los que fueron procesados estadísticamente utilizando el programa informatizado Stadistical Program for Social Science (SPSS) versión 17 para Windows.

Los resultados del análisis estadístico fueron interpretados y discutidos en función del marco teórico y la literatura pertinente: Finalmente se elaboraron conclusiones. 


\section{RESULTADOS}

A continuación se exponen los resultados alcanzados.

Los datos de la tabla 1 indican que en los resultados de componentes del lenguaje, no existen diferencias significativas en el componente fonéticofonológico ( $(38)=0.78, p>.05)$, aunque se aprecia que los niños con pérdida auditiva prelocutiva que utilizan implante coclear obtuvieron una puntuación promedio ligeramente menor $(\mathrm{M}=$ $65.10, \mathrm{DE}=2.92$ ) que la alcanzada por los niños oyentes $(\mathrm{M}=66.30, \mathrm{DE}=5.32)$.

En lo que corresponde al componente morfológico se encuentra que existen diferencias significativas entre los puntajes obtenidos en el componente morfológico ( $\mathrm{t}(38)=5.23, \mathrm{p}<.05)$. Observándose, además, que el rendimiento promedio del grupo de niños con implante coclear $(\mathrm{M}=7.30, \mathrm{DE}=7.17)$ es menor en el componente morfológico respecto al grupo oyente $(\mathrm{M}=18.73$, $\mathrm{DE}=9.10$ ).

Con relación al componente sintáctico, se puede apreciar que no existen diferencias significativas entre los puntajes obtenidos en el componente sintáctico entre (38) $=1.91, \mathrm{p}>.05)$, aunque los niños oyentes alcanzan un mejor rendimiento $(\mathrm{M}=18.70, \mathrm{DE}=8.18)$ con relación a los niños con pérdida auditiva $(\mathrm{M}=13.70, \mathrm{DE}=7.51)$.

En el componente semántico se observa que existen diferencias significativas ( $\mathrm{t}(38)=2.50, \mathrm{p}<.05)$ apreciándose que el mejor desempeño corresponde a los niños oyentes $(\mathrm{M}=14.67, \mathrm{DE}=3.27)$ frente a los niños con implante coclear $(\mathrm{M}=12.30, \mathrm{DE}=3.02)$.

En lo que respecta al componente pragmático se halla que existen diferencias significativas entre los puntajes obtenidos en el componente pragmático $((38)=4.18, \mathrm{p}<.05)$, apreciándose que el desempeño de los niños con implante coclear $(\mathrm{M}=10.00, \mathrm{DE}=6.55)$ es menor que el de los niños oyentes $(\mathrm{M}=17.47$, $\mathrm{DE}=6.14$ ).

Finalmente, en el puntaje total del lenguaje, se encuentra que existen diferencias significativas en los puntajes totales del lenguaje ( $\mathrm{t}(38)=3.83, \mathrm{p}<.05)$, apreciándose que el puntaje promedio obtenido por los niños oyentes es mayor $(\mathrm{M}=119.40, \mathrm{DE}=16.98)$ que el alcanzado por los niños con pérdida auditiva prelocutiva con implante coclear $(\mathrm{M}=$ 99.40, $\mathrm{DE}=13.37$ ). 
Tabla 1

Análisis comparativo del nivel de los componentes del lenguaje ponente

\begin{tabular}{lccccc}
\hline \multirow{2}{*}{$\begin{array}{c}\text { Componentes } \\
\text { del lenguaje }\end{array}$} & \multicolumn{2}{c}{$\begin{array}{c}\text { Niños con } \\
\text { implante coclear }\end{array}$} & \multicolumn{2}{c}{ Niños oyentes } & \multirow{2}{*}{$\mathbf{t}$} \\
\cline { 2 - 5 } & $\mathbf{M}$ & $\mathbf{D E}$ & $\mathbf{M}$ & $\mathbf{D E}$ & \\
Fonética-fonología & 65.10 & 2.92 & 66.30 & 5.32 & 0.78 \\
Morfología & 7.30 & 7.17 & 18.73 & 9.10 & $5.23^{*}$ \\
Sintaxis & 13.70 & 7.51 & 18.70 & 8.18 & 1.91 \\
Léxico-semántica & 12.30 & 3.02 & 14.67 & 3.27 & $2.50^{*}$ \\
Pragmática & 10.00 & 6.55 & 17.47 & 6.14 & $4.18^{*}$ \\
Total & 99.40 & 16.99 & 119.40 & 13.37 & 3.83 * \\
\hline
\end{tabular}

${ }^{*} p<.05$

Nota: 10 niños tuvieron implante coclear y 30 fueron oyentes.

\section{DISCUSIÓN}

El lenguaje ha sido valorado en función de las puntuaciones otorgadas a los niños por cada acierto obtenido. Al analizar dichos resultados, tanto del grupo de niños oyentes como de los niños con implante coclear, se encuentran diferencias significativas a favor del grupo de niños oyentes; sin embargo, esta superioridad no se da en todos los aspectos. Observamos entonces que en tres de cinco aspectos evaluados los niños oyentes han obtenido mejores resultados, asumimos que esta diferencia se debe a que los niños que utilizan implante coclear y que han sido educados bajo una metodología auditiva oral se han visto favorecidos por esta en el desarrollo de las dimensiones de forma del lenguaje, vale decir el componente fonético-fonológico y el componente sintáctico, pero no en el componente morfológico y en las dimensiones de contenido y uso, donde presentan dificultades. Lo hallado guarda relación con lo expuesto por Furmanski (2003), quien concluyó que la terapia auditiva verbal es un tipo de abordaje muy recomendable para los niños pequeños, señalando que esta metodología se ajusta perfectamente a los requerimientos de un niño con implante, dados sus fundamentos y principios.

En el componente fonéticofonológico no se hallaron diferencias entre los grupos observados, esto se debe al trabajo de discriminación auditiva, lo cual corresponde a la metodología auditiva-oral y que ha favorecido a los niños con pérdida auditiva prelocutiva que utilizan implante coclear que les ha permitido alcanzar un nivel 
de desempeño muy similar al de los niños oyentes. Lo mismo se observa al analizar el componente sintáctico, el que guarda relación con el trabajo de sintaxis de la metodología ya mencionada, a la que han estado expuestos los niños con pérdida auditiva prelocutiva que utilizan implante coclear, que han logrado una adecuada organización de las oraciones utilizadas, llegando a un nivel de desempeño similar al de los niños oyentes. Lo hallado se encuentra en línea con lo expuesto por Franco, Tabanez, Bevilacqua y Vuono (2007), quienes concluyeron que el lenguaje de los niños que usan implante coclear es semejante al de los niños oyentes de 5 años de edad para la expresión y semejante al de los niños de 4 años para la comprensión.

Por otro lado, se encontraron diferencias en la dimensión de contenido del lenguaje correspondiente al componente léxico-semántico, el cual analiza la palabra en su máxima dimensión, desarrollando no solo el significado sino considerando también los morfemas de esta. En la evaluación de este componente se puede observar que las respuestas de los niños oyentes fueron cortas y concisas, respondiendo solo a la solicitud de la examinadora independientemente de que la respuesta sea correcta, mientras que los niños con pérdida auditiva prelocutiva intentaron en todo momento explicar la situación observada en la lámina, más allá de poder realizar la tarea solicitada e in- dependientemente de que la respuesta sea correcta o no. Todo lo expuesto se encuentra en correspondencia con lo hallado por Rondal (Acosta \& Moreno, 1999), ellos señalan que a partir de los 6 años el desarrollo del lenguaje es muy particular tanto para la expresión como para la comprensión y depende de numerosas variables, como la socialización, la escolarización, etcétera.

Los niños con pérdida auditiva prelocutiva que utilizan implante coclear han logrado un promedio de desarrollo de lenguaje ligeramente menor al alcanzado por el grupo de niños oyentes, esta diferencia se encuentra respaldada por lo propuesto por Sharman, Dorman y Spahr (Furmanski, 2003), quienes afirman que existe un periodo en los primeros años de vida durante el cual el sistema nervioso central conserva su máxima plasticidad, por ello los niños implantados en edad preescolar emplean la audición para la adquisición del lenguaje, monitorean sus producciones auditivamente, su habla es muy inteligible y el progreso en el lenguaje tiene un ritmo similar al de los niños con audición normal. Ello respalda la necesidad de considerar a los profesionales, a la familia del niño y las variables intrínsecas del niño implantado, como los factores que intervienen en su rehabilitación.

Szagun (2006) tenía por objetivo estudiar el desarrollo del lenguaje en 22 niños con pérdida auditiva neurosensorial profunda bilateral que utilizan 
implante coclear. Este fue un estudio a largo plazo, que se realizó entre 1996 y 2000, concluyó que los niños sordos que reciben un implante coclear a temprana edad son capaces de alcanzar el balbuceo canónico en menos tiempo del que los niños con audición normal suelen hacerlo y que la adquisición de los fonemas sigue la misma secuencia que la de sus pares con audición normal. Estos resultados son consistentes con la literatura que indica que la identificación y la intervención temprana son importantes para permitir a los niños con implante coclear la oportunidad de estar al mismo nivel que sus compañeros de audición normal. Esta información respalda lo hallado en el desarrollo fonético fonológico en la muestra de niños con pérdida auditiva prelocutiva que utilizan implante coclear, quienes alcanzaron un desarrollo de este componente del lenguaje muy similar al logrado por los niños oyentes.

Los resultados no solo nos permitieron conocer el nivel de desarrollo del lenguaje que presentan tanto los niños oyentes como los niños con pérdida auditiva prelocutiva que utilizan implante coclear, sino conocer el desempeño de cada niño. Se apreció que ambos grupos presentan dificultades en los niveles morfológico y pragmático, ello nos lleva a inferir que el desempeño apreciado por los niños evaluados depende en parte de los factores descritos en cada grupo, pero aun dentro de los mismos grupos la variabilidad en los resultados es grande y podría atribuirse a factores cognitivos relacionados con el aprendizaje, la memoria, la atención y el procesamiento del lenguaje.

Por otro lado, los logros en habilidades de percepción auditiva y habilidades comunicativas apreciadas en los niños con pérdida auditiva prelocutiva que utilizan implante coclear varían enormemente de sujeto a sujeto, sin embargo es evidente que todos los sujetos de nuestro estudio lograron beneficios en mayor o menor grado; notamos entonces lo importante que resulta habilitar el canal auditivo para la percepción de los sonidos del habla y cómo esto repercute en la comunicación y, como consecuencia, en su rendimiento académico, su inserción social y su calidad de vida. Esto guarda relación con lo expuesto por Furmanski (2003), en la medida en que su desempeño con implante en las áreas correspondientes a la audición y la comunicación sea adecuado, se podrán esperar resultados similares en los demás aspectos a los que se hace referencia.

En base a lo expuesto, se acepta la hipótesis general, ya que los resultados respaldan la existencia de diferencias en el lenguaje en los niños con pérdida auditiva prelocutiva que utilizan implante coclear y los niños oyentes de 7 a 10 años de edad. 


\section{ConCLUSIONES}

A partir de los resultados, se concluye lo siguiente:

- El nivel de lenguaje del niño oyente mostró una curva de desarrollo adecuado, observándose ciertas dificultades en los componentes correspondientes a la morfológica y la pragmática.

- El nivel de lenguaje del niño con pérdida auditiva prelocutiva que utiliza implante coclear muestra una curva de desarrollo de los componentes aun en proceso.

- El nivel de lenguaje de los niños oyentes es sustancialmente mayor al nivel de lenguaje de los niños con pérdida auditiva prelocutiva que utilizan implante coclear. En el uso de componente fonético-fonológico no se hallaron diferencias significativas entre los niños con pérdida auditiva prelocutiva que utilizan implante coclear y los niños oyentes de 7 a 10 años de edad.

- Existen diferencias significativas entre los puntajes obtenidos en el componente léxico-semántico entre los niños con pérdida auditiva prelocutiva que utilizan implante coclear y los niños oyentes de 7 a 10 años de edad.

- Existen diferencias significativas entre los puntajes obtenidos en el componente morfológico entre los niños con pérdida auditiva prelocutiva que utilizan implante coclear y los niños oyentes de 7 a 10 años de edad.

- No existen diferencias significativas entre los puntajes obtenidos en el componente sintáctico entre los niños con pérdida auditiva prelocutiva que utilizan implante coclear $\mathrm{y}$ los niños oyentes de 7 a 10 años de edad.

- Existen diferencias significativas entre los puntajes obtenidos en el componente pragmático entre los niños con pérdida auditiva prelocutiva que utilizan implante coclear y los niños oyentes de 7 a 10 años de edad.

\section{REFERENCIAS}

Acosta, V., \& Moreno, A. (1999). Dificultades del lenguaje en ambientes educativos. Del trastorno especifico del lenguaje. Barcelona: Masson.

Aguado, G. (1995). Desarrollo del lenguaje de 0 a 3 años. Madrid: CEPE.

Berrocal, N. (s/f). Deficiencia auditiva en el niño. Aspectos sociolingüisticos y pedagógicos. Portal del Ministerio de Educación. Recuperado de http://portal.perueduca.edu.pe/basicaespecial/articulos.htm

Castañeda, P. (1999). El lenguaje verbal del niño sordo. Lima: Universidad Nacional Mayor de San Marcos.

Davis, H., \& Silverman, S. (1971). Audición y sordera. México, D. F.: La Prensa Médica Mexicana. 
Franco, R., Tabanez, L., Bevilacqua, M., \& Vuono, R. (2007). Lenguaje oral en niños con cinco años de uso de implante coclear. Pro-Fono. Revista de Actualización Cientifica, 19(2). Recuperado de http://www.scielo.br/ pdf/pfono/v19n2/en_a05v19n2.pdf

Furmanski, H. (2003). Implantes cocleares en niños. (Re)habilitación auditiva y terapia auditivo-verbal. Madrid: Nexus ediciones.

López, M. (2005). La educación de las personas con sordera. La escuela oralista española. Valencia: Universidad de Valencia, Servicio de Publicaciones.

Manrique, M. \& Huarte, A. (2000). La sordera. Etiología, tipos y soluciones. En: II Jornadas de Logopedia. Patologías del Desarrollo del Lenguaje y Medidas Restauradoras. Sevilla.
Martínez, E. (1998). Lingüística: teoría y aplicaciones. Barcelona: Masson.

Mortari, A., Bevilacqua, M. \& Alves, G. (2007). Desempeño de la audición y del lenguaje oral en niños con deficiencia auditiva neurosensorial profunda pre-lingual que usan implante coclear. Revista de Actualización Científica 19(3), 295-304. Recuperado de www.scielo.com

Narbona, J. \& Chevrie-Muller, C. (2001). El lenguaje del niño. (2. ${ }^{a}$ edición). Barcelona: Masson.

Silvestre, N. (2003). Sordera, comunicación y aprendizaje. Barcelona: Else-Masson.

Szagun, G. (2006). Desarrollo del lenguaje en niños con implante coclear. Recuperado de www.cultura-sorda.eu 